\title{
Size-Exclusion Chromatography of Carboxyl-terminated Dendrimers as a Model for Permeation of Charged Particles into Like-Charged Cavities
}

\author{
G. Shah, ${ }^{*}$ P. L. Dubin, ${ }^{*}, 1$ J. I. Kaplan, $\dagger$ G. R. Newkome, $\ddagger$ C. N. Moorefield $\ddagger$ And G. R. BakeR $\ddagger$ \\ * Department of Chemistry, Indiana University-Purdue University, Indianapolis, Indiana 46202; and †Department of Physics \\ and $\ddagger$ Department of Chemistry, University of South Florida, Tampa, Florida 33620
}

Received February 16, 1996; accepted May 15, 1996

\begin{abstract}
In order to examine the permeation of small charged colloids into cavities of like charge, size-exclusion chromatography was carried out with carboxyl-terminated dendritic polymers on a porous glass stationary phase. Chromatographic partition coefficients $K_{\mathrm{SEC}}$ were measured for solute diameters ranging from 2 to $8 \mathrm{~nm}$, at neutral $\mathrm{pH}$, and ionic strengths from 0.01 to $0.09 \mathrm{M}$, and were reported relative to those of noninteracting solutes of equal size (pullulan and Ficoll). The experimental results were compared to values calculated using the treatment of F. G. Smith III and W. M. Deen for charged spheres in cylindrical pores of like charge (J. Colloid Interface Sci. 78, 44 (1980); 91, 571 (1983)). The measured degrees of permeation were typically $20-100 \%$ larger than the calculated values, the discrepancies being greatest for the larger solutes at high ionic strength. It is shown that the tendency of the calculations to overestimate the repulsion are likely to arise from linearization of the Poisson - B oltzmann equation. (c) 1996 A cademic Press, Inc.
\end{abstract}

\section{INTRODUCTION}

The permeation of macromolecular solutes into porous materials is a factor in such diverse fields as ultrafiltration, renal transport, enhanced oil recovery, and chromatography. In aqueous systems, most porous materials acquire a surface charge, usually negative, either because of adsorption of small ions or because of intrinsic ionophores. If the macromolecular species are similarly charged, repulsive effects will reduce their permeation. Several manifestations of this effect may be noted. The restricted diffusion of macroions through porous membranes with similar charge is strongly dependent on ionic strength (1). The permeation of polyelectrolytes into porous glass substrates, as measured by size exclusion chromatography (SEC), is sensitive to both ionic strength and $\mathrm{pH}$, the latter influencing the charge on the glass stationary phase $(2-5)$. Similar results have been noted for anionic charged latexes chromatographed on porous glass

\footnotetext{
${ }^{1}$ To whom correspondence should be addressed.
}

(6) or silica $(7,8)$. SEC partition coefficients of proteins on polymer-based chromatographic gels also display clear evidence of repulsive effects when $\mathrm{pH}$ is larger than the protein isoelectric points $(9,10)$.

While there are numerous qualitative reports of the diminution of permeation by electrostatic repulsion, quantitative treatments are less common. Lin and Deen (11) measured the diffusivity of polystyrene sulfonate through track-etched nucleopore membranes as a function of ionic strength and membrane pore radius, and compared the data to theoretical results obtained from a model that incorporates long-range solute-pore interactions into the partitioning of flexible chain macromolecules in like-charged pores (12). The reduction in diffusivity with decrease in ionic strength could be attributed to a reduction in the partition coefficient related to an increase in the Debye length. The measured ratio of pore diffusion to free diffusion $\left(D / D_{\infty}\right)$ was found to be in general agreement with predictions from theoretical results. However, the situation was complicated because it was necessary to calculate the polymer charge taking into account counterion condensation and because the polymer dimensions (radius of gyration) at different ionic strengths were obtained via extrapolation of literature data. With regard to the latter problem, treatment of rigid solutes should offer some simplification. Hoagland, for example, studied the permeation of charged rods into cavities of like charge (13). Smith and Deen $(14,15)$ provided a theoretical treatment for the permeation of spherical solutes (uniform surface charge, or uniform volume charge) into cylindrical pores; comparison with measurements for hindered diffusion of either ficoll sulfate (a densely branched polyanion) or dextran sulfate (a weakly branched polyanion) yielded some rather large discrepancies (1), and it was suggested that these differences might reflect the choice of the hydrodynamic model.

The foregoing discussion is intended to suggest the value of carrying out measurements with a solute that behaves as a well-defined surface-charged sphere. Furthermore, SEC offers, in at least one sense, a better test of permeation theo- 
ries than restricted diffusion experiments, inasmuch as the hydrodynamic effects (enhanced drag within the pore) do not need to be considered (16). This approach depends, of course, on the availability of an SEC stationary phase/solute system that is well characterized with respect to geometry and charge. Latexes on porous silica or glass might seem to offer such a system, but we and others (6) have observed strong adsorption of latexes on siliceous packings. Dendrimers provide an interesting class of compounds for this reason, since solute structure appears to be well defined, with charge groups residing at the distal ends of the molecule which may then constitute a "charged surface." We have recently discussed the merit of carboxyl-terminated dendrimers as SEC standards (17); however, in that work we attempted to suppress electrostatic effects by appropriate choice of ionic strength and $\mathrm{pH}$. Under conditions of lower ionic strength, and higher $\mathrm{pH}$, carboxyl-terminated dendrimers would seem to provide a good test of models for the restricted permeation of surface-charged spheres into like-charge cavities, which is the theme of the current work.

In this work we focus on the treatment of Smith and Deen $(14,15)$ in which the permeation coefficient of a charged sphere into a like-charged cylindrical cavity is computed as a function of the sphere and cylinder radii, the cylindrical surface charge density, the ionic strength, and the sphere charge. The S\&D theory considers spheres of either uniform surface charge density or uniform volume charge density. The dendritic polymers $(18,19)$ used here must conform to the former case, since the charges are located on the distal segments. As a reference material, we use the electrically neutral macromolecule Ficoll, which is densely branched and so may be considered as an uncharged sphere (20). The selection of an appropriate stationary phase is problematic. Ideally, this material would have cylindrical pores of narrow size distribution, with well-defined surfaces whose surface charge densities may be readily determined. No commercial

TABLE 1 Characteristics of Pullulan Standards

\begin{tabular}{lrcrrrr}
\hline Sample & $M_{\mathrm{w}}{ }^{a}$ & $\bar{M}_{\mathrm{w}} / \bar{M}_{\mathrm{n}}{ }^{a}$ & $\begin{array}{c}{[\eta]^{b}} \\
\left(\mathrm{~cm}^{3} / \mathrm{g}\right)\end{array}$ & $\begin{array}{c}R_{\eta} \\
(\AA)^{c}\end{array}$ & $\begin{array}{c}{[\eta]^{d}} \\
\left(\mathrm{~cm}^{3} / \mathrm{g}\right)\end{array}$ & $\begin{array}{c}R_{\eta} \\
(\AA)^{e}\end{array}$ \\
\hline P-800 & 853,000 & 1.14 & 187.0 & 294 & 171 & 285 \\
P-200 & 186,000 & 1.13 & 70.4 & 128 & 55 & 118 \\
P-100 & 100,000 & 1.10 & 45.9 & 90 & 39.8 & 86 \\
P-50 & 48,000 & 1.09 & 28.6 & 60 & 23.4 & 56 \\
P-20 & 23,700 & 1.07 & 18.1 & 41 & 15.5 & 39 \\
P-10 & 12,200 & 1.06 & 11.9 & 28 & 9.7 & 27 \\
P-5 & 5,800 & 1.07 & 7.9 & 19 & 6.3 & 18 \\
\hline
\end{tabular}

\footnotetext{
${ }^{a}$ Supplied by the manufacturer.

${ }^{b}$ In water, at $25^{\circ} \mathrm{C}$ (supplied by the manufacturer).

${ }^{c}$ From columns two and four via Eq. [6].

${ }^{d}$ From Ref. (10), in $0.2 \mathrm{M}$ sodium phosphate buffer (pH 7.0) at $25^{\circ} \mathrm{C}$.

${ }^{e}$ From columns two and six via Eq. [6].
}

TABLE 2

Characteristics of Ficoll Fractions ${ }^{a}$

\begin{tabular}{lrrc}
\hline \multicolumn{1}{c}{ Fraction } & $M_{\mathrm{w}}$ & $M_{\mathrm{n}}$ & $M_{\mathrm{w}} / M_{\mathrm{n}}$ \\
\hline T1800, from (9) & 714,000 & 337,000 & 2.1 \\
T1800, from (12) & 461,000 & 257,000 & 1.8 \\
T1800, from (15) & 321,000 & 244,000 & 1.3 \\
T1800, from (20) & 132,000 & 113,700 & 1.2 \\
T2580-IVB, from (2) & 71,800 & 64,600 & 1.1 \\
T2580-IVB, from (11) & 21,800 & 20,300 & 1.1 \\
\hline
\end{tabular}

${ }^{a}$ From supplier.

material fulfills all these requirements. We have selected crushed porous glass as the optimal available stationary phase. Developed by Haller (21), "CPG'" is prepared by careful heat treatment which develops heterogeneous regions; after crushing and sieving, the softer phase is removed by acid leaching. In contrast to packings based on synthetic polymers or polysaccharides, porous glass can be characterized by mercury intrusion. The materials prepared by Haller typically had narrow pore size distributions (PSD) of 515\% (22); commercial CPG may have larger PSDs but still narrow compared to many semirigid gels. The pore structure of CPG as seen by SEM has been compared to swiss cheese (23). Nevertheless, results obtained with various solutes conform closely to a linear dependence of the square root of the chromatographic partition coefficient (permeation coefficient) on solute radii, which is the behavior predicted for cylindrical pores $(4,24)$.

\section{EXPERIMENTAL \\ Materials}

Narrow molecular weight distribution (MWD) standards of the linear nonionic polysaccharide pullulan, described in Table 1 (P-82, Lot. 10101), were purchased from SDK Showa Denko (New York, NY). MW fractions of the densely branched nonionic polysaccharide ficoll were gifts from Dr. Kristi Granath of Kabi Pharmacia, Uppsala. Fractions 1-4 were characterized by SEC on Sepharose 4B and 5-6 on Sephadex G-200 Superfine. The characteristics of the fractions are described in Table 2. Cascade polymers (Table 3) were prepared according to the procedure of Young et al. (18) and fully characterized. The hydrodynamic radii were calculated via the Stokes-Einstein equation from measured diffusion coefficients obtained with pulsed field gradient NMR for each polymer generation at about $1 \mathrm{mM}$ concentration (19). Sodium phosphate, $\mathrm{NaCl}, \mathrm{HCl}, \mathrm{NaOH}$, and $\mathrm{D}_{2} \mathrm{O}$ were of reagent grade. Milli-Q water was used throughout the experiment.

\section{Methods}

\section{(i) Size Exclusion Chromatography (SEC)}

The chromatography system consisted of a Milton Roy Mini Pump (St. Petersburg, FL), a $75-\mu \mathrm{L}$ injection loop 
TABLE 3

Characteristics of $\mathrm{C}$ ascade Polymers

\begin{tabular}{ccrc}
\hline Generation & $\begin{array}{c}\text { Number of terminal } \\
\text { COOH }\end{array}$ & MW & $\begin{array}{c}\text { Hydrodynamic } \\
\text { radius }(\AA)^{a}\end{array}$ \\
\hline 1 & 12 & 1,341 & 12.3 \\
2 & 36 & 4,092 & 17.3 \\
3 & 108 & 12,345 & 23.9 \\
4 & 324 & 37,102 & 33.1 \\
5 & 972 & 111,373 & 37.1 \\
\hline
\end{tabular}

${ }^{a}$ From Ref. (18), at neutral pH.

from Valco Instruments (Houston, TX), an R401 differential refractometer from Waters Associates (Milford, MA), and an Omniscribe Recorder from Houston Instruments (Austin, TX). Stainless steel columns $(50 \times 0.55 \mathrm{~cm}$ i.d. $)$ with 2$\mu \mathrm{m}$ end frits from Supelco (Bellefonte, PA) were dry packed with BIORAN-CPG from Schott Gerate (Mainz, Germany). The column efficiency was obtained by injecting $10 \%$ of $\mathrm{D}_{2} \mathrm{O}$ in $I=0.003 \mathrm{M}, \mathrm{pH} 7.0$ sodium phosphate buffer on CPG-1. Characteristics of the CPG columns are shown in Table 4.

Mobile phases were prepared from $\mathrm{NaH}_{2} \mathrm{PO}_{4}$ and $\mathrm{Na}_{2} \mathrm{HPO}_{4}$ to the required ionic strength and $\mathrm{pH}$, then filtered through $0.45-\mu \mathrm{m}$ filters. Chromatograms were obtained by injecting 3 to $5 \mathrm{mg} / \mathrm{mL}$ of polymer solution at a flow rate of 0.8 to $0.9 \mathrm{~mL} / \mathrm{min}$ and chart speed of $0.5 \mathrm{~mL} / \mathrm{min}$. Elution volumes were obtained using flow rates which were measured periodically by weighing the column eluant, respectively. $V_{\mathrm{t}}$, total volume, and $V_{0}$, void volume, were obtained by injecting $\mathrm{D}_{2} \mathrm{O}$ and pullulan 800 , respectively. The chromatographic partition coefficient was determined as $K_{\mathrm{SEC}}=$ $\left(V_{\mathrm{e}}-V_{0}\right) /\left(V_{\mathrm{t}}-V_{0}\right)$.

\section{(ii) Measurement of Surface Charge Density of Packing}

$\mathrm{pH}$ measurements of CPG were performed with an Orion 811 research $\mathrm{pH}$ meter (Boston, MA), with a Beckmann combination electrode. About $0.05 \mathrm{~g}$ of CPG-1 was weighed precisely into a glass vial with a stir bar, and exactly 12.00 $\mathrm{g}$ of $0.01 M \mathrm{NaCl}$ was added. After passing $\mathrm{N}_{2}$ through the solution for $5-10 \mathrm{~min}$, the $\mathrm{pH}$ was adjusted to 7.00 by adding less than $0.01 \mathrm{~mL}$ of $0.2 \mathrm{M} \mathrm{NaOH}$. The solution was titrated with $0.05 \mathrm{M} \mathrm{HCl}$ to $\mathrm{pH} 3.0$ under $\mathrm{N}_{2}$ atmosphere with a 0.2 mL Gilmont microburette (Great Neck, NY). The amount of titrant was adjusted to get nearly $0.1 \mathrm{pH}$ increments. A blank of $12.00 \mathrm{~g}$ of $0.01 \mathrm{M} \mathrm{NaCl}$ was titrated in close proximity to the sample. The difference in titrant volume for sample and blank was calculated at corresponding $\mathrm{pH}$ 's during the titration. The titration was concluded when the difference between $V_{\mathrm{s}}$ and $V_{\mathrm{b}}$ became constant, at which point the solute would be fully protonated (zero charge).

A similar titration was carried out from $\mathrm{pH} 7.00$ to 10.00 using $0.02 M \mathrm{NaOH}$. Since constant $\left(V_{\mathrm{s}}-V_{\mathrm{b}}\right)$ was not observed up to $\mathrm{pH} 10.00$, the sample still has some $-\mathrm{SiOH}$ groups at this $\mathrm{pH}$. The amount of $\mathrm{NaOH}$ that would be required to bring a fully protonated sample to a specified $\mathrm{pH} \leqslant 7.0$ was calculated as $\left(V_{\mathrm{s}}-V_{\mathrm{b}}\right)_{0}-\left(V_{\mathrm{s}}-V_{\mathrm{b}}\right)$, where $\left(V_{\mathrm{s}}-V_{\mathrm{b}}\right)_{0}$ corresponds to the lower endpoint. For $\mathrm{pH}>$ 7.0 , the corresponding quantity was $V_{\mathrm{s}}-V_{\mathrm{b}}$, where $V$ is the volume of added base. Multiplication by the molarity of titrant gave the number of milliequivalents of $\mathrm{SiO}^{-}$formed, $n$. The packing surface charge density was calculated as

$$
\left.\sigma_{\mathrm{p}}=9[n / 1000] N_{\mathrm{A}}\right) / m \cdot e \cdot S
$$

where $N_{\mathrm{A}}$ is Avogadro's number, $e$ is the elementary charge in coulombs, $m$ is the weight of the sample in grams, and $S$ is the specific surface area of sample in $\mathrm{m}^{2} / \mathrm{g}$. A similar procedure was applied to determine the surface charge density at 0.03 and $0.05 \mathrm{M} \mathrm{NaCl}$.

\section{(iii) Measurement of Surface Charge Density of Solutes}

A. Structural method. To determine the number of $-\mathrm{COO}^{-} /$ molecule at any $\mathrm{pH}$, cascade polymers were titrated in a manner similar to the packing material. Solutions containing $3 \mathrm{mg}$ of dendrimer were prepared in $100 \mathrm{~mL}$ of $0.01,0.03$, and $0.05 \mathrm{M} \mathrm{NaCl}$. Titrations were carried out as described in (ii) above, with the substitution of 0.02 for $0.05 \mathrm{M} \mathrm{NaOH}$. The formal surface charge density, $\sigma_{\mathrm{s}}$, can be determined from the number of $-\mathrm{COO}^{-}$so that

$$
\sigma_{\mathrm{s}}=\left[M_{\mathrm{b}}\left(V_{\mathrm{s}}-V_{\mathrm{b}}\right) / 1000\right] \times \mathrm{MW} / m\left[e / 4 \pi R_{\eta}^{2}\right]
$$

where $n$ is the number of moles of $\mathrm{COO}^{-}$formed, $M_{\mathrm{b}}$ is the molarity of titrant, $V_{\mathrm{s}}$ is the volume of titrant (in milliliters) for the sample, $V_{\mathrm{b}}$ is the volume of titrant (in milliliters) for the blank, MW is molecular weight of the polymer, $m$ is the mass of the sample used for titration, $e$ is the elementary charge (in coulombs), and $R_{\eta}$ is the viscosity radius (in meters). The surface charge density, $\sigma_{\mathrm{s}}\left(\mathrm{C} \mathrm{m}^{-2}\right)$, obtained in this way was called the structural charge density.

B. Potential method. The titration curves yield also $\alpha$, the degree of ionization, and thus $\mathrm{p} K_{\mathrm{a}}=\mathrm{pH}+\log [(1-$ $\alpha) / \alpha]$. The surface potential $\phi_{0}$ may be obtained as

\section{TABLE 4} Characterization of $\mathrm{CPG}^{a}$

\begin{tabular}{lcccc}
\hline & $\begin{array}{c}\text { Grain size } \\
(\mu \mathrm{m})^{a}\end{array}$ & $\begin{array}{c}\text { Por radius } \\
(\AA)^{b}\end{array}$ & $\begin{array}{c}\text { Specific surface } \\
\text { area }\left(\mathrm{m}^{2} / \mathrm{g}\right)\end{array}$ & Theoretical plates $^{c}$ \\
\hline CPG-1 & $30-60$ & 130 & 136 & 1000 \\
\hline
\end{tabular}

${ }^{a}$ Supplied by manufacturer.

${ }^{b}$ Measured by mercury porosimetry (supplied by manufacturer).

${ }^{c}$ Experimentally measured in $50 \mathrm{~cm} \times 0.55 \mathrm{~cm}$ i.d. packed column. 


$$
\mathrm{p} K_{\mathrm{a}}-\mathrm{p} K_{0}=0.434\left(e \phi_{0} / k T\right)
$$

where $\mathrm{p} K_{0}$ is $\mathrm{p} K_{\mathrm{a}}$ extrapolated to $\alpha=0$. The relevant "surface" is the mean locus of bound protons.

For spherical particles of radius $r$, the surface charge density, $\sigma_{\varphi}$, may be obtained from $\phi_{0}(25)$

$$
\sigma_{\varphi}=\phi_{0} \varepsilon_{r} \varepsilon_{0}(1+\kappa r) / r
$$

where $\varepsilon_{r}$ is the dielectric constant (at $25^{\circ} \mathrm{C}$ ), $\varepsilon_{0}$ is the permitivity of vacuum $\left(\mathrm{F} \mathrm{m}^{-1}\right)$, and $\kappa\left(\mathrm{m}^{-1}\right)$ is the reciprocal Debye length (all in MKS units, so that $\sigma_{\varphi}$ has units of $\mathrm{C} \mathrm{m}^{-2}$ ).

\section{(iv) Electrophoretic Light Scattering}

The electrophoretic mobility of the generation number 5 cascade polymer was measured on a Coulter Delsa 440 (Langley Ford Instruments, Amherst, MA) using a $\sim 7 \mathrm{mg}$ / mL solution in $10 \mathrm{mM}$ pH 7 sodium phosphate buffer. The relationship between zeta potential, $\zeta$, and electrophoretic mobility, $u(26)$, is

$$
u=c \zeta \varepsilon_{r} \varepsilon_{0} / \eta
$$

where $\varepsilon_{r}=78.54$ at $25^{\circ} \mathrm{C}, \varepsilon_{0}=8.85 \times 10^{-12} \mathrm{~F} \mathrm{~m}^{-1}, c$ is a dimensionless constant, and $\eta$, the viscosity, $=0.8902 \times$ $10^{-3} \mathrm{~N} \mathrm{~s} \mathrm{~m}^{-2}$ at $25^{\circ} \mathrm{C}$. The value of $c$ for $\kappa R<0.1$ is $2 / 3$ while for $\kappa R>100, c$ is 1 . As $\kappa R$ was about $2, c=2 / 3$ was used in our case.

\section{(v) Viscosity}

Intrinsic viscosities of Ficoll fractions were measured with a Schott Gerate AVS-400 equipped with a 2-mL capacity capillary viscometer (capillary constant $=0.008352$ ) at $25.0 \pm$ $0.02^{\circ} \mathrm{C}$. The Ficoll samples were dissolved in water or $I=0.2$ $M$, pH 4.5 sodium phosphate buffer, at concentrations ranging from 10 to $24 \mathrm{mg} / \mathrm{mL}$. All samples were filtered through $0.45-$

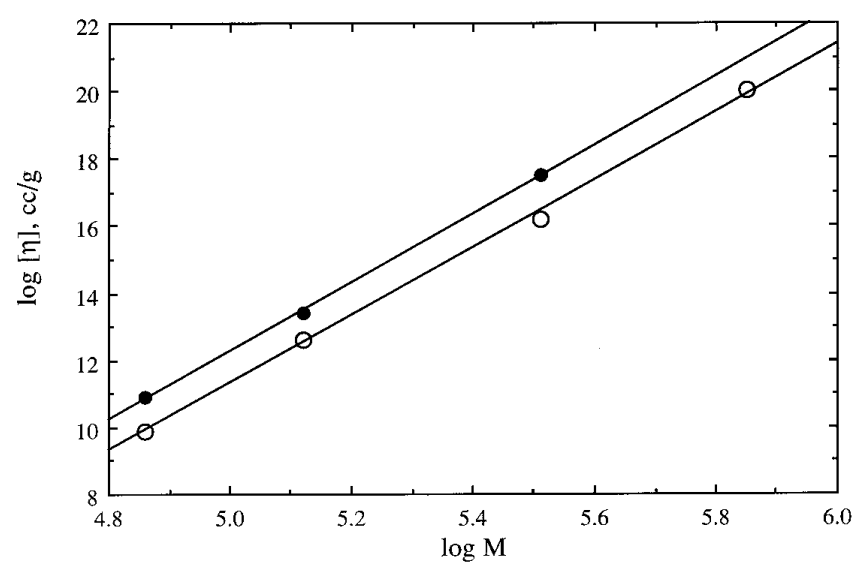

FIG . 1. Mark-Houwink plots for Ficoll in pure water $(\bigcirc)$, and $0.3 \mathrm{M}$, $\mathrm{pH} 4.5$ phosphate buffer $(\bullet)$.
TABLE 5

Size Measurements of Ficoll Fractions

\begin{tabular}{lccccr}
\hline \multicolumn{1}{c}{ Fraction } & $\bar{M}_{\mathrm{w}}{ }^{a}$ & $\begin{array}{c}{[\eta]^{b}} \\
\left(\mathrm{~cm}^{3} / \mathrm{g}\right)\end{array}$ & $\begin{array}{c}R_{\eta}{ }^{c} \\
(\AA)\end{array}$ & $\begin{array}{c}{[\eta]^{d}} \\
\left(\mathrm{~cm}^{3} / \mathrm{g}\right)\end{array}$ & $\begin{array}{r}R_{\eta}{ }^{e} \\
(\AA)\end{array}$ \\
\hline T1800, Fr. 9 & 714,000 & 20 & 131 & $23.9^{f}$ & 139 \\
T1800, Fr. 12 & 461,000 & $17.5^{f}$ & 109 & $20.8^{f}$ & 115 \\
T1800, Fr. 15 & 321,000 & 16.2 & 94 & 17.5 & 96 \\
T1800, Fr. 20 & 132,000 & 12.6 & 64 & 13.4 & 65 \\
T2580-IVB, Fr. 2 & 71,800 & 9.9 & 48 & 10.9 & 50 \\
T2580-IVB, Fr. 11 & 21,800 & $7.0^{f}$ & 29 & $7.8^{f}$ & 30 \\
\hline
\end{tabular}

${ }^{a}$ From supplier.

${ }^{b}$ In water at $25^{\circ} \mathrm{C}$.

${ }^{c}$ From column 2 and 3, via Eq. (6).

${ }^{d}$ In $0.2 \mathrm{M}$ sodium phosphate buffer $(\mathrm{pH} 4.5)$ at $25^{\circ} \mathrm{C}$.

${ }^{e}$ From column 2 and 5, via Eq. (6).

${ }^{f}$ Interpolated value.

$\mu \mathrm{m}$ Gelman filters. Efflux times, in the range of 107-113 s, were measured with a precision of $\pm 0.02 \mathrm{~s}$. The intrinsic viscosity, $[\eta]\left(\mathrm{cm}^{3} / \mathrm{g}\right)$, was obtained from the intercept of the plot of $\eta_{\mathrm{sp}} / c$ vs $c$, where $\eta_{\mathrm{sp}}$ is $\left(\eta_{\text {rel }}-1\right)$, and $\eta_{\text {rel }}$ is the ratio of efflux time of the pure solvent to the sample solution. $R_{\eta}$ (cm) can be calculated from the equation (27)

$$
R_{\eta}=\left(\frac{3[\eta] M}{10 \pi N_{\mathrm{A}}}\right)^{1 / 3},
$$

where $M$ is the molecular weight of the polymer.

Viscosities for cascade polymers were measured in an identical manner. In order to avoid aggregation, $12-15 \mathrm{mg} /$ $\mathrm{mL}$ solutions of the polymers in water were adjusted to $\mathrm{pH}$ 7 with the addition of $0.07-0.12 \mathrm{~mL} 1 \mathrm{~N} \mathrm{NaOH}$ per $1 \mathrm{~mL}$ of sample solution.

\section{(vi) Conductivity}

Ionic strengths of the dendrimer solutions used for viscometry were determined by conductimetry at $25^{\circ} \mathrm{C}$. The resistance of cascade polymer solutions was measured after 25- to 50-fold dilution, depending on the amount available. A calibration curve was obtained by measuring the resistance of sodium chloride solutions of known ionic strength (conductivity $=$ cell constant $/$ resistance $)$ and used to determine the apparent ionic strength of the dendrimer solution.

\section{(vii) Calculations}

All calculations were based on equations from Ref. (15), which were solved using Mathematica (Addison-Wesley).

\section{RESULTS AND DISCUSSION}

\section{Characterization of Ficolls}

Intrinsic viscosities were measured for Ficoll fractions in water and in $0.3 \mathrm{M}, \mathrm{pH} 4.5$ phosphate buffer at $25 \pm 0.02^{\circ} \mathrm{C}$. 
The results are presented in the double logarithmic plots of Fig. 1, and conform to the Mark-Houwink relation $[\eta]=$ $K M^{a}$ with $K, a$ equal to $0.30,0.35$ in water, and $0.32,0.32$ in buffer. The former result is in moderate agreement with Lavrenko (20) et al. who found $K, a=0.2,0.35$ in water at $26^{\circ} \mathrm{C}$. The enhancement in Ficoll viscosity with ionic strength was observed also by Shah and Dubin (28) who found an elevation of $15 \%$ in $[\eta]$ for $7 \times 10^{5}$ MW Ficoll upon raising the ionic strength from zero to $0.1 \mathrm{M}$ sodium phosphate. Viscosity radii calculated from $[\eta]$ and MW are shown in Table 5. The ionic-strength dependence of the Ficoll radii is very small.

\section{Characterization of C ascade Polymers}

The structure of G2 cascade polymer, shown in Fig. 2, indicates that these solutes can be treated to a close approximation as spheres. In this case, various hydrodynamic methods should yield the same radius. Because of the high preci- sion of intrinsic viscosity measurements, even for relatively small solutes, we elected to determine the viscosity radius. Direct dissolution of cascade polymers in $0.01 \mathrm{M}, \mathrm{pH} 7.00$ phosphate buffer, produced a drop of $3 \mathrm{pH}$ units, due to exchange of $\mathrm{Na}^{+}$for $\mathrm{H}^{+}$from the polymer. We therefore chose to adjust the $\mathrm{pH}$ of polymer in pure water by the addition of $\mathrm{NaOH}$, and then to determine the ionic strength conductimetrically. The relationship between ionic strength and conductivity was obtained by measuring the conductivity of $\mathrm{NaCl}$ solutions from 0.05 to $5 \mathrm{mM}$. Application of this calibration to the measured conductivities of cascade polymers at $\mathrm{pH} 7$ produced the results shown in Fig. 3. The relatively low slopes for G3 and G5 can be explained in terms of the relatively low degree of ionization for these solutes at any $\mathrm{pH}$, which was also observed by potentiometric titration (see below). Figure 4 shows the concentration dependence of the reduced specific viscosity in pure water (after $\mathrm{pH}$ adjustment with $\mathrm{NaOH}$ ) plotted according to the empirical equation put forward by Fuoss $(29),\left(\eta_{\mathrm{sp}} / c\right)^{-1}=$

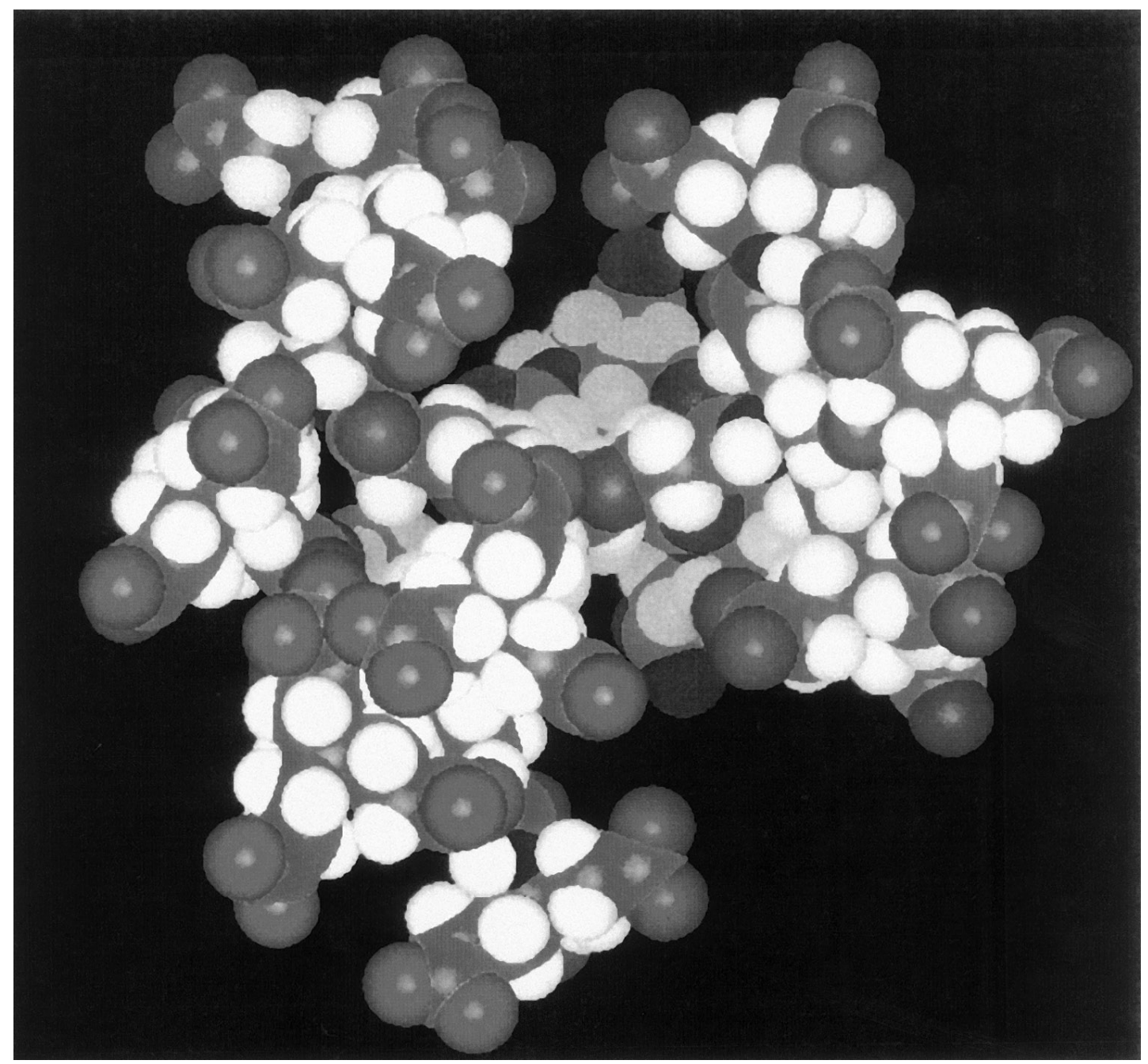

FIG. 2. Structure of $G=2$ cascade polymer. 


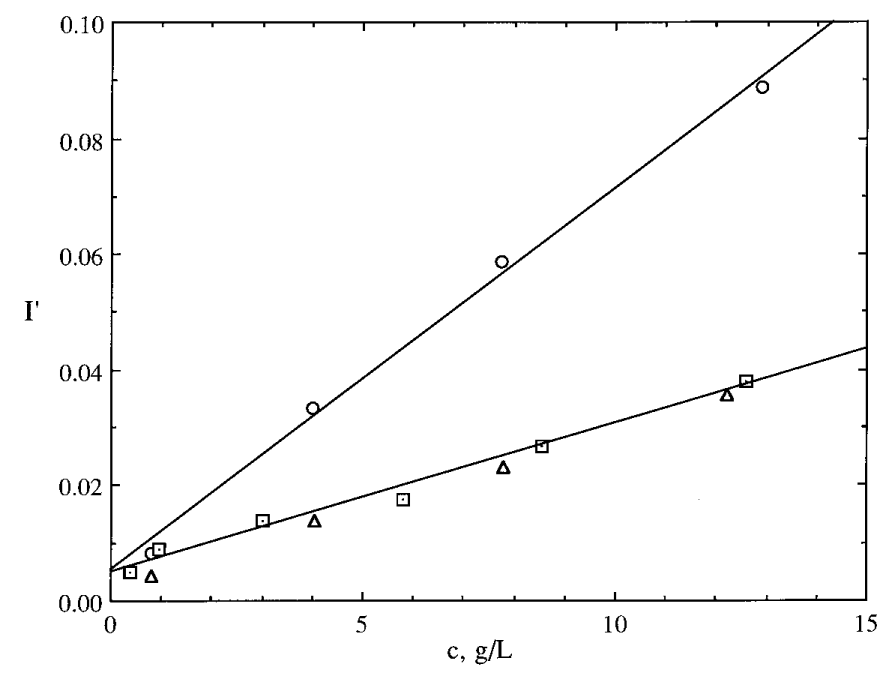

FIG. 3. Apparent ionic strength (from conductivity) in pure water for cascade polymers G1 $(\bigcirc)$, G3 $(\triangle)$, and G5 $(\square)$.

$A+B c^{1 / 2}$. However, we observed nonlinear plots of $c / \eta_{\text {sp }}$ vs $c^{1 / 2}$, which might be due to the very high polymer concentration and its effect on the ionic strength. On the basis of the curves in Figs. 2 and 3, we determined $\eta_{\mathrm{sp}} / c$ for each polymer at ionic strengths of $0.01,0.03$, and 0.05 , and thence the apparent viscosity radii $R_{\eta}^{\prime}$, where the prime indicates a value based on $\eta_{\mathrm{sp}} / c$ instead of [ $\eta$ ]. These values are reported for the three cascade polymers at different ionic strengths in Table 6.

$\mathrm{pH}$ titration curves are presented in Figs. 5-7 as the number of carboxylate groups formed per molecule vs $\mathrm{pH}$, at each of three ionic strengths. Titration showed a well-defined high-pH endpoint at which the number of $\mathrm{COO}^{-} /$molecule was within $\pm 10 \%$ of the calculated value. These data enable us to determine a "structural charge density," $\sigma_{\mathrm{s}}$. As ex-

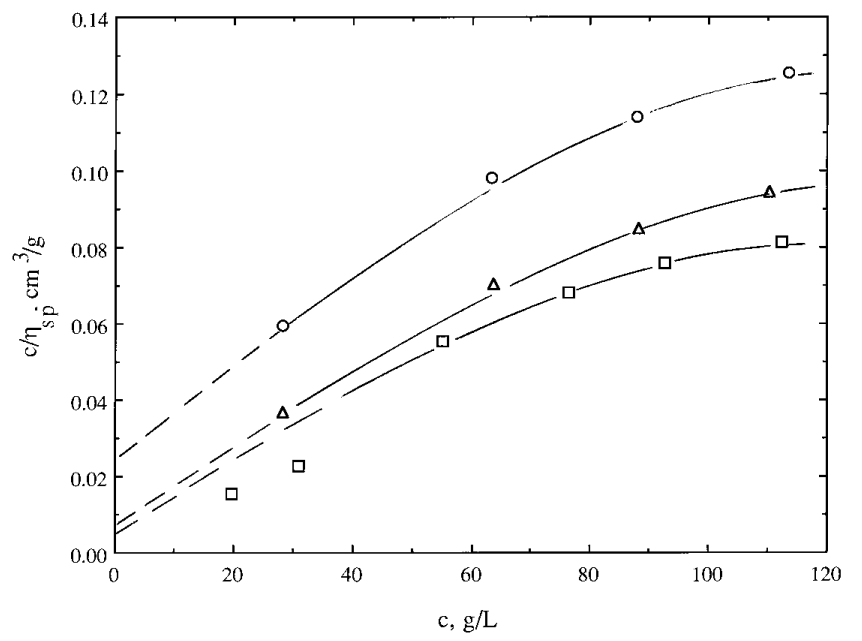

FIG . 4. Concentration dependence of reduced specific viscosity in pure water, for G1 $(\bigcirc)$, G3 $(\square)$, and G5 $(\square)$, plotted according to Fuoss equation.
TABLE 6

Surface C harge Density of Cascade Polymers at $\mathrm{pH}=\mathbf{7 . 0}$

\begin{tabular}{cccccc}
\hline $\begin{array}{c}\text { Generation } \\
\text { number }\end{array}$ & $\begin{array}{c}\text { Apparent ionic } \\
\text { strength, I', (M) }\end{array}$ & $\begin{array}{c}\phi_{0}{ }^{a} \\
(\text { volt })\end{array}$ & $\begin{array}{c}R_{\eta}{ }^{b} \\
(\AA)\end{array}$ & $\begin{array}{c}\sigma_{\phi}{ }^{c} \\
\left(\mathrm{C} \mathrm{m}^{-2}\right)\end{array}$ & $\begin{array}{c}\sigma_{\mathrm{s}}{ }^{d} \\
\left(\mathrm{C} \mathrm{m}^{-2}\right)\end{array}$ \\
\hline \multirow{2}{*}{1} & 0.01 & 0.055 & 15 & 0.038 & 0.062 \\
& 0.03 & 0.045 & 14 & 0.041 & 0.084 \\
& 0.05 & 0.047 & 13 & 0.050 & 0.090 \\
3 & 0.01 & 0.051 & 32 & 0.018 & 0.085 \\
& 0.03 & 0.041 & 28 & 0.033 & 0.142 \\
& 0.05 & 0.046 & 26 & 0.036 & 0.161 \\
5 & 0.01 & 0.051 & 85 & 0.016 & 0.018 \\
& 0.03 & 0.049 & 62 & 0.025 & 0.267 \\
& 0.05 & 0.048 & 58 & 0.030 & 0.284 \\
\hline
\end{tabular}

${ }^{a}$ Surface potential from potentiometric titration.

${ }^{b}$ From viscosity measurement.

${ }^{c}$ Surface charge density calculated using Eq. [4] and column 3.

${ }^{d}$ Surface charge density calculated using Eq. [2] and column 4.

pected from electrostatic considerations, the polymers are weaker acids at the lowest ionic strength. The titration data can also be analyzed as plots of $\mathrm{p} K_{\mathrm{a}}=\mathrm{pH}+\log [(1-\alpha) /$ $\alpha$ ] vs $\alpha$, with a representative example shown in Fig. 8 for G-5. From this plot one may determine the surface potential, $\phi_{0}$, via Eq. [3], and thence the surface charge density, using Eq. [4]. The surface charge density determined in this way, $\sigma_{\phi}$, is given in Table 6 , along with $\sigma_{\mathrm{s}}$ and $\phi_{0}$. The values of $\sigma_{\phi}$ are significantly larger than the values of $\sigma_{\mathrm{s}}$. Electrophoretic mobility was carried out on G5 in $10 \mathrm{mM}, \mathrm{pH} 7$ buffer and, by application of Eq. [4], yielded a zeta potential of $48 \mathrm{mV}$, in excellent agreement with $\phi_{0}$. We therefore conclude that the potentiometric method provides the correct surface potential, and that the structural surface charge density is too large because it neglects counterion binding. Structural charge densities in general increase with generation number due to closer packing of ionophores, while po-

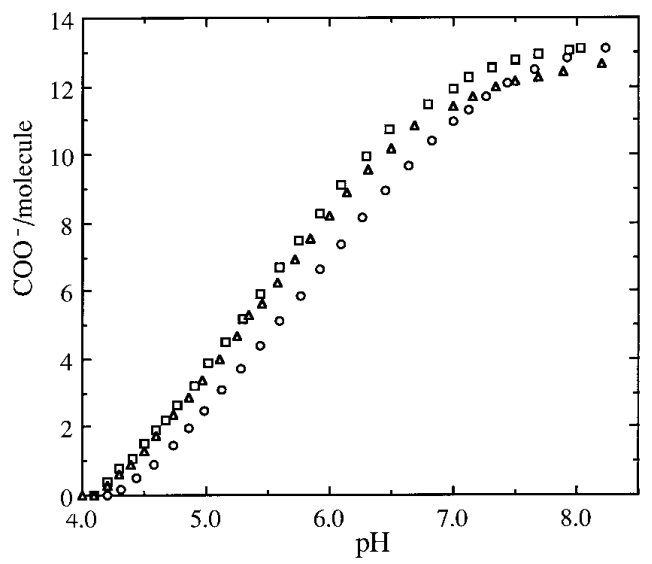

FIG . 5. pH titration curves presented as number of $\mathrm{COO}^{-}$per molecule for $\mathrm{G} 1$, at ionic strengths $0.01(\bigcirc), 0.03(\square)$, and $0.05 M(\triangle)$. 


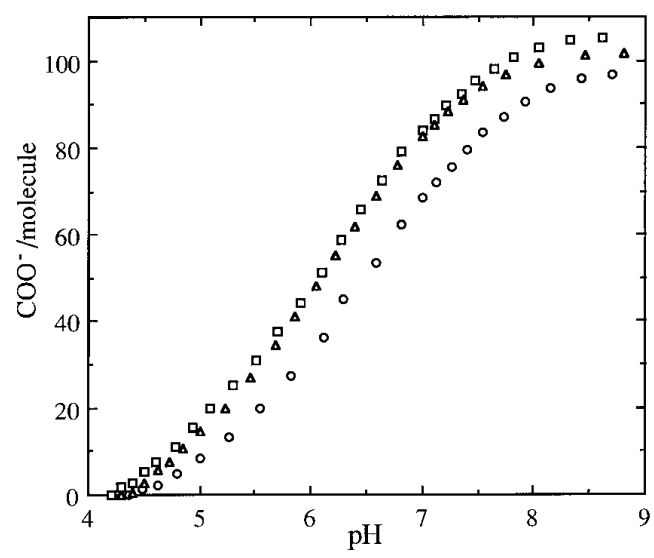

FIG. 6. pH titration curves presented as number of $\mathrm{COO}^{-}$per molecule for $\mathrm{G} 3$, at ionic strengths $0.01(\bigcirc), 0.03(\square)$, and $0.05 M(\triangle)$.

tentiometric surface charge densities decrease with increasing generation number. This may be attributable to the pronounced difference in counterion binding between spherical and planar charged surfaces (30).

\section{Characterization of Porous G lass}

Chromatographic partition coefficients of Ficoll and pullulan are plotted vs viscosity radii in $3 \mathrm{mM}$, pH 7.0 buffer, in Fig. 9. Nine of the 12 data fall on a line corresponding to the equation $K_{\mathrm{SEC}}=\left[\left(r_{\mathrm{p}}-R_{\eta}\right) / r_{\mathrm{p}}\right]^{2}$ which describes the permeation of a sphere of radius $R_{\eta}$ into a cylindrical cavity of radius $r_{\mathrm{p}}$. The negative reciprocal of the slope in Fig. 9 then gives the mean pore radius as $140 \AA$, in good agreement with the manufacturer's porosimetry result of $130 \AA$. The good linearity seen in Fig. 9 is also evidence of narrow pore size distribution: if the PSD were significant, then at low $K$ values the polymers would permeate only the larger pores, the apparent pore size would increase, and the slope would change (22).

The surface charge density of CPG $\sigma_{\mathrm{p}}$ was obtained by $\mathrm{pH}$ titration, with the results shown in Fig. 10. As expected, the glass is more acidic at higher ionic strength. From the titration curves, the surface charge density of the glass at $\mathrm{pH} 7$ was calculated at ionic strengths of 10,30 , and 50 $\mathrm{m} M$, as $0.09 \mathrm{C}, 0.019 \mathrm{C}$, and $0.021 \mathrm{C} \mathrm{m}^{-2}$, respectively.

\section{Chromatography}

Values of $K_{\mathrm{SEC}}$ for G1-G5 cascade polymers, measured in $\mathrm{pH}$ 6.0, 7.0, and 8.0 phosphate buffers, ranging from 10 to $200 \mathrm{mM}$, are shown in Table 7. As expected, permeation increases with increased ionic strength, with decreasing $\mathrm{pH}$, and with decreasing solute size. The variation of $K_{\mathrm{SEC}}$ with $\mathrm{pH}$, at $50 \mathrm{mM}$, is much larger for G5 than for G1. In part, this is attributable to the strong repulsion of G5, but it is also a reflection of adsorption of G5 (with $K_{\mathrm{SEC}}$ larger than

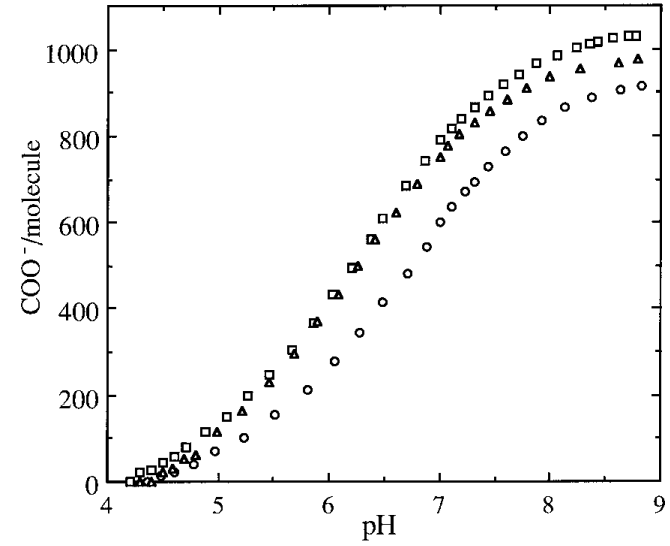

FIG. 7. pH titration curves presented as number of $\mathrm{COO}^{-}$per molecule for G5, at ionic strengths $0.01(\bigcirc), 0.03(\square)$, and $0.05 M(\triangle)$.

all the other polymers at $\mathrm{pH} 6.0$ and $I=50 \mathrm{mM}$, despite its greater size).

We would like to evaluate the extent of repulsion of the cascade polymers by comparison with the "calibration", curve for Ficoll and pullulan, as shown in Fig. 11 for G1, $\mathrm{G} 3$, and $\mathrm{G} 5$ at $\mathrm{pH}$ 7.0. Note, however, that the data point for G5 in $90 \mathrm{~m} M$ buffer crosses the Ficoll/pullulan curve, indicating adsorption. This becomes more evident at $\mathrm{pH}$ 6.0, as seen in Fig. 12. The adsorption of G5 at higher ionic strength and lower $\mathrm{pH}$ can be understood by the increase in $\mathrm{COOH}$ and $\mathrm{SiOH}$ groups on polymer and glass, respectively, accompanied by a decrease in repulsive forces. The increased planarity of the two surfaces also contributes to hydrogen bonding between the two species. Such adsorptive interactions are even more prominent with other colloidal solutes. We found that a 7-nm "carboxyl-modified latex," which eluted in the exclusion limit in $3 \mathrm{mM} \mathrm{pH} 7$ buffer, was completely adsorbed in $90 \mathrm{~m} M$ buffer. Similarly, 6- and 11-nm Ludox samples (HS40 and TM, respectively) were

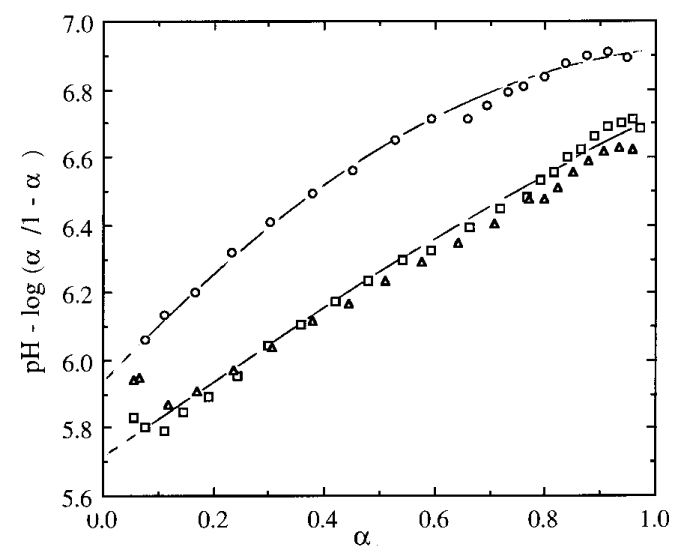

FIG. 8. Dependence of $\mathrm{p} K_{\mathrm{a}}$ on $\alpha$ for G5, in $0.01 M$ (Fig. 5. $\mathrm{pH}$ titration curves presented as number of $\mathrm{COO}^{-}$per molecule for $\mathrm{G} 1$, at ionic strengths $0.01(\bigcirc), 0.03(\square)$, and $0.05 M(\triangle)$. 


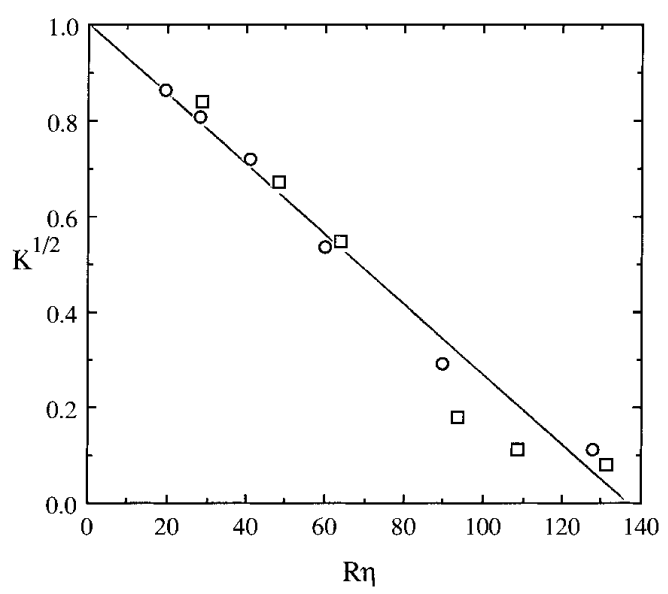

FIG. 9. Dependence of $K_{\mathrm{SEC}}$ on solute size for pullulan $(\bigcirc)$ and Ficoll ( $\square$ ) in $0.003 M$, pH 7.0 phosphate buffer.

completely excluded in $3 \mathrm{~m} M \mathrm{pH} 7$ buffer, but completely adsorbed in $200 \mathrm{~m} M$ buffer.

Elimination of adsorptive mechanisms from consideration poses a problem. However, we note that several workers have observed a linear dependence between $R_{\text {eff }}$ and $I^{-1 / 2}$, where $R_{\text {eff }}$ is the radius of the neutral polymer with the same $K_{\mathrm{SEC}}$ as the charged solute. This dependence has been seen for latexes on CPG (8), for proteins at $\mathrm{pH}>\mathrm{pI}$ on $\mathrm{PW}$ gel (31) and for charged Ficoll fractions on Superose (32), all situations in which both solute and packing are negatively charged. Figure 13 confirms this dependence for G1 and G3, but reveals negative deviations (retardation on the column) for G3 and G5 at the highest ionic strength. It seems reasonable to conclude that only measurements carried out with G3 and G5 at $I>100 \mathrm{~m} M$ are subject to adsorption.

Since the cascade polymers are nearly spherical and the charges are on the periphery, we chose to evaluate the constant surface charge density model of Ref. (15). The interaction energy $E(\beta)$, which depends on $\sigma_{\varphi}, \sigma_{\mathrm{p}}, \kappa, r_{\mathrm{p}}$, and $R_{\eta}$, was obtained using Eq. [39] of Ref. (15), since all the variables have been experimentally determined. The partition coefficient was then obtained by solving Eq. [9] of Ref. (15)

$$
K=2 \int_{0}^{1-\alpha} \exp [-E(\beta) / k T] \beta d \beta
$$

Here, $\beta$ is a dimensionless parameter that describes the position of a charged sphere within a like-charged cylinder. All calculations were done using Mathematica. In order to compare experimental and theoretical evaluations of the repulsion effect, we report $\Delta K_{0}$, the calculated difference between $K$ of the charged solute and a neutral solute of the same size, obtained via Eq. [7] for each cascade polymer under each condition. $\Delta K_{0}$ is then compared to the measured $\Delta K_{\mathrm{SEC}}$ obtained from Fig. 11 . Note that $\Delta K_{0} / \Delta K_{\mathrm{SEC}}=\ln$
$\Delta G_{\text {el }}-\ln \Delta G_{\text {exp }}$, where $\ln \Delta G_{\text {el }}$ is the calculated electrostatic free energy from Ref. (15), and $\Delta G_{\text {exp }}$ contains all nonideal contributions (not necessarily limited to electrostatic) to permeation. The results are shown in Table 8 . The agreement is good for G1 and G3 at the lowest ionic strengths, but becomes progressively worse at higher ionic strength or higher generation number. In all cases, the extent of permeation appears to be underestimated by theory. Indeed, with the exception of $\mathrm{G} 1$ in 30 and $50 \mathrm{mM}$ buffer, and $\mathrm{G} 2$ in $50 \mathrm{~m} M$ buffer, calculated values of $K$ are always less than 0.15 , whereas experimental values (see Table 7) are never lower than 0.20 , except for $I=10 \mathrm{~m} M$.

A number of explanations could be offered for the disagreement between the measured exclusion effect and the theoretical prediction. The theoretical value could be in error due to errors in the various parameters used in the calculation, as follows: (a) In using the ionic strength of the medium in the calculation, we assume no contribution of the cascade polymer to the ionic strength. (b) The structural charge density of the CPG obtained by potentiometric titration could be higher than the true value because Stern layer effects are not considered; i.e., it is assumed that only $\mathrm{H}^{+}$, but not $\mathrm{Na}^{+}$, can neutralize $\mathrm{SiO}^{-}$(the surface charge density of the cascade polymer was calculated from the measured surface potential which does include Stern layer effects). (c) While the pore size we measured is in good agreement with the manufacturer's mercury porosimetry data, we did not consider the effect of the pore size distribution. (d) To calculate the radii of the cascade polymers, $\eta_{\mathrm{sp}} / \mathrm{c}$ was used instead of $[\eta]$. (e) Adsorption of the solute on the glass could contribute a nonelectrostatic, nonsteric component to $K_{\mathrm{SEC}}$, making these values too large.

The influence of these errors can be discussed as follows: (a) If the contribution of cascade polymer concentration

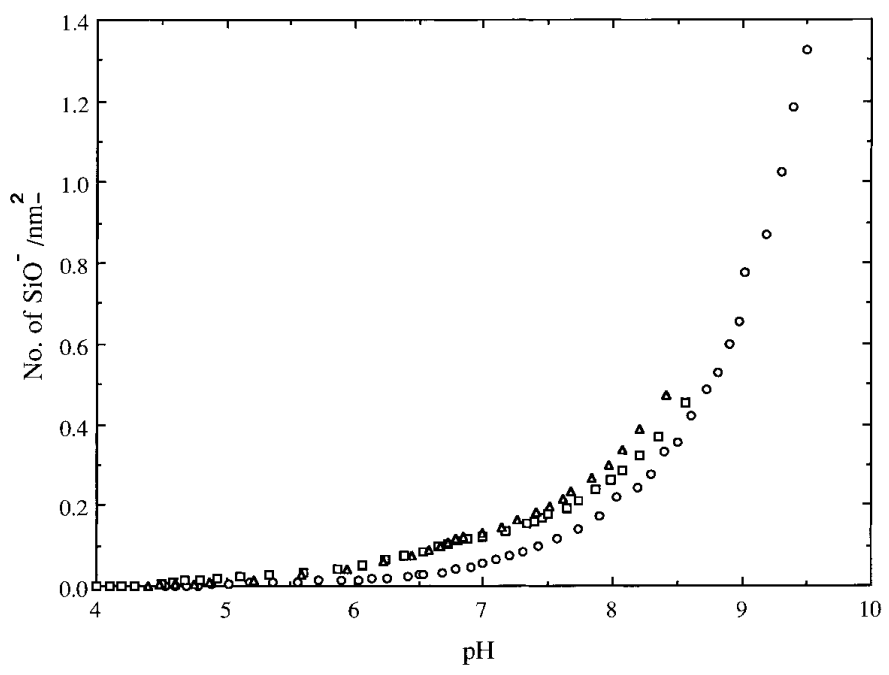

FIG . 10. Dependence of CPG surface charge on $\mathrm{pH}$ for $0.01(\bigcirc), 0.03$ $(\square)$, and $0.05 M(\triangle) \mathrm{NaCl}$. 
TABLE 7

$\mathrm{K}_{\mathrm{SEC}}$ of Cascade Polymers in Sodium Phosphate Buffer at pH 7.0

\begin{tabular}{cccccccc}
\hline $\mathrm{G}$ & $I=0.01 M$ & $I=0.03 M$ & $I=0.05 M$ & $I=0.09 M$ & $I=0.2 M$ & $I=0.05 M^{a}$ & $I=0.05 M^{b}$ \\
\hline 1 & 0.20 & 0.45 & 0.56 & 0.61 & 0.76 & 0.69 & 0.51 \\
2 & 0.12 & 0.36 & 0.48 & 0.54 & 0.71 & 0.63 & 0.41 \\
3 & 0.06 & 0.30 & 0.42 & 0.49 & 0.68 & 0.81 & 0.35 \\
4 & 0.04 & 0.22 & 0.37 & 0.45 & 0.64 & 0.89 & 0.28 \\
5 & 0.03 & 0.20 & 0.32 & 0.39 & 0.60 & 0.94 & 0.26 \\
\hline
\end{tabular}

${ }^{a} \mathrm{pH} 6.0$.

${ }^{b} \mathrm{pH} 8.0$.

relative to the ionic strength were considered, the resulting ionic strength would be slightly larger, reducing $\Delta K$ (theoretical) and giving better agreement. (b) Consideration of Stern layer effects would provide a smaller CPG potential, leading to a lower estimate of the repulsion effect (better agreement). (c) The effect of pore size distribution is complicated, but unlikely to be large compared to the observed discrepancy. (d) Substitution of $\eta_{\mathrm{sp}} / \mathrm{c}$ for $[\eta]$ changes the radius by $3 \%$ which is too small to matter.

The effects mentioned in (a) and (b) would be greatest at low ionic strength, but this is where the best agreement is observed. As for (c) and (d) the magnitudes of these effects are too small to be significant. Adsorption of solute (e) is more problematic, especially as adsorption effects would be expected to increase with ionic strength. Potschka (9) has rationalized retention data for proteins that are partially electrostatically excluded from the packing by

$$
R_{\mathrm{eff}}=R+x \kappa^{-1}
$$

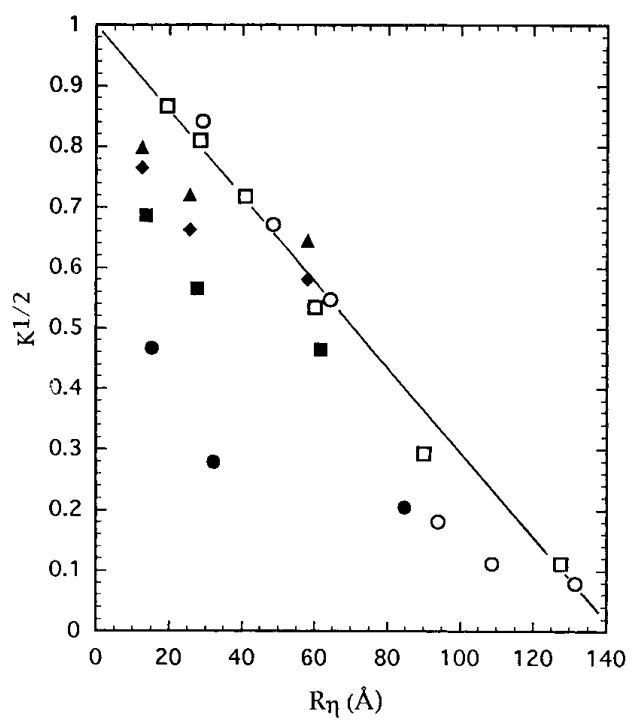

FIG. 11. Dependence of chromatographic partition coefficient on solute radius for pullulan $(\square)$ and Ficoll $(\bigcirc)$ in $0.003 M$, pH 7.0 phosphate buffer, and cascade polymers in pH 7 buffer at ionic strengths $0.01(\bullet), 0.03(\boldsymbol{\square})$, $0.05(\diamond)$, and $0.09 M(\boldsymbol{\Delta})$. where $R_{\text {eff }}$ is the "apparent size" of the solute, from the observed retention volume compared to those of solutes that do not interact with the packing, $R$ is the geometric size of the solute, and $x$ is the average electrostatic repulsion distance in multiples of Debye lengths. When the retention volumes for the cascade polymers at $\mathrm{pH} 8$ are plotted in Fig. 13 according to Eq. [8], a linear dependence of $R_{\text {eff }}$ on $I^{-1 / 2}\left(\sim \kappa^{-1}\right)$ is observed, which is not consistent with the enhancement of adsorption with increasing ionic strength.

If the values of $K_{\mathrm{SEC}}, \kappa, R, r, \sigma_{\varphi}$, and $\sigma_{\mathrm{s}}$ are correct, then it is necessary to consider limitations of the theory. The most prominent of these is the use of the linearized form of the Poisson-Boltzmann equation (PBE), as is done in Ref. (15), which could lead to the underestimation of the extent of permeation. Appendix 1 shows that the linearized form of the PBE always gives a larger value for the surface potential than does the nonlinear form. It is instructive to estimate the magnitude of this effect. Let us consider a typical set of conditions, with

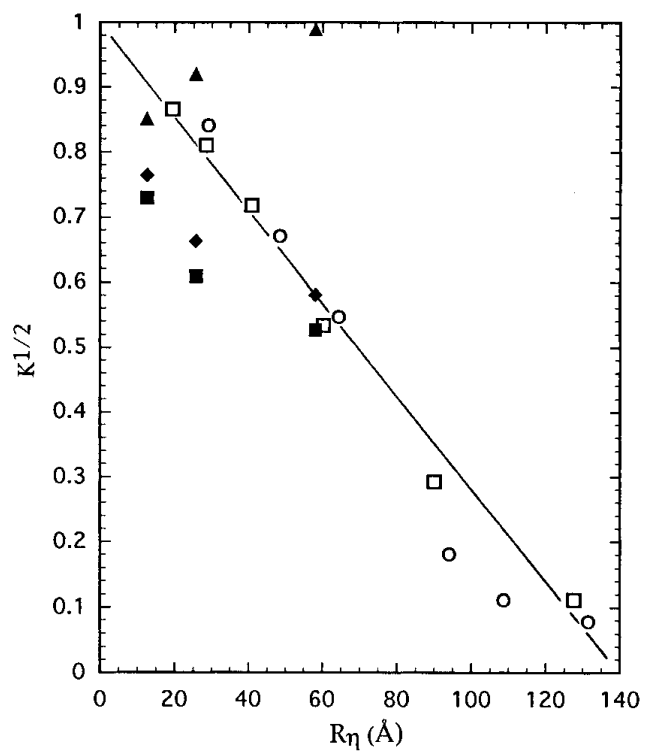

FIG . 12. Dependence of chromatographic partition coefficient on solute radius for pullulan $(\square)$ and Ficoll $(\bigcirc)$ in $0.003 M$, pH 7.0 phosphate buffer, and cascade polymers in $0.05 \mathrm{M}$ buffer at $\mathrm{pH} 8.0(\boldsymbol{\square}), 7.0(\diamond)$, and 6.0 $(\boldsymbol{\Delta})$. 


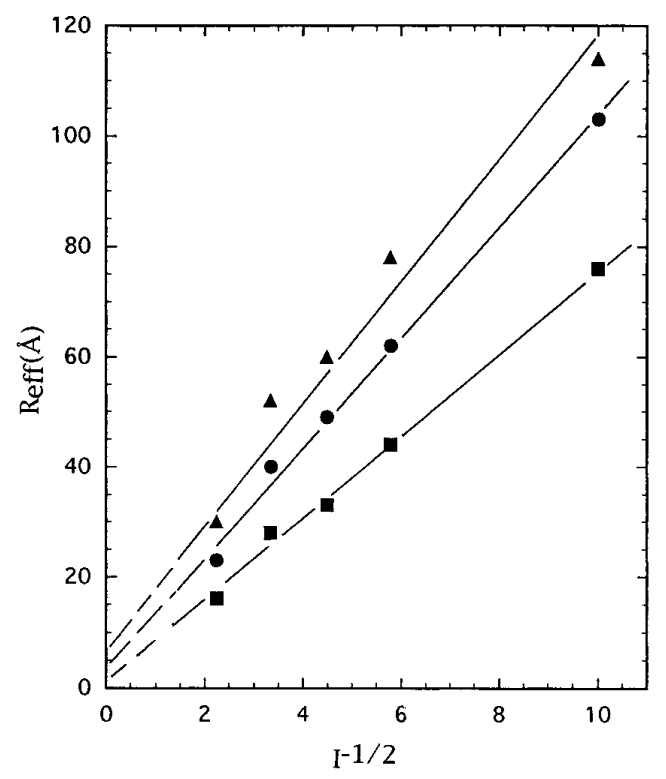

FIG. 13. Ionic strength dependence of effective radius at $\mathrm{pH} 7.0$ for

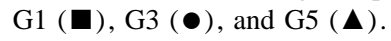

the surface charge density on the cylindrical cavity at $0.02 \mathrm{C} /$ $\mathrm{m}^{2}$ and the surface charge density on the sphere at $0.03 \mathrm{C} / \mathrm{m}^{2}$, at an ionic strength of $0.05 \mathrm{M}$, and ambient temperature $\left(300^{\circ} \mathrm{K}\right)$. At a distance $z_{0}$ between the sphere and the cylinder such that $z_{0} \kappa=1$, the potential for the cylinder is found to be $\phi=4 k T / e$ with the linearized PBE, and $\phi=2.1 k T / e$ with the nonlinearized form. As a result, the repulsive force is significantly overestimated, and permeation is underestimated. The approximation is most severe in the case of high solute surface potentials, namely for higher generation dendrimers. Thus, the deviations between experiment and theory expected from use of a linearized PBE are of the same direction and magnitude as what we observe. Furthermore, it seems likely that the effect of linearization is sufficient in magnitude to account for the differences seen in Table 8. More rigorous attempts to modify the approach of Ref. (15) are beyond the scope of the current work.

\section{CONCLUSIONS}

The permeation of spherical charged particles into cavities of similar charge shows poor agreement with the theory of
Smith and Deen (15), which consistently overestimates the repulsive force. It seems most likely that this discrepancy arises from the use of the linearized form of the PoissonBoltzmann equation. This linearization is often considered to be justified when the surface potentials involved are "small compared to $k T$." While "small compared to" is inherently vague, there is no doubt that this assumption is incorrect for the dendrimer surface potentials, which are on the order of $50 \mathrm{mV}$, i.e., almost twice as large as $k T / e$. It seems likely that particles whose surface potentials were small enough to satisfy the linearization restriction would display reductions in permeation too small to measure.

\section{APPENDIX 1}

The solution of the Poisson-Boltzmann equation

$$
\nabla^{2} \phi=2 n_{0} q \sinh (q \phi / k T)
$$

for fixed surface charge density on the cylinder and on the sphere is solved subject to the boundary condition that the normal derivative of the potential at either the cylinder surface or the surface of the sphere is equal to the surface charge at the respective surfaces divided by the effective dielectric constant, i.e.,

$$
\frac{d \phi}{d n}=\sigma / \varepsilon \varepsilon_{0}
$$

for either cylinder or sphere surface. The linearized form of Eq. [A1] is given as

$$
\nabla^{2} \phi_{\mathrm{L}}=2 n_{0} q\left(q \phi_{\mathrm{L}} / k T\right)
$$

A one-dimensional representation of the problem, i.e., $d \phi /$ $d n=d \phi / d z$ appears as shown below

\begin{tabular}{|c|c|c|c|c|c|c|c|c|c|}
\hline \multirow[b]{2}{*}{ G } & \multicolumn{3}{|c|}{$0.01 M$} & \multicolumn{3}{|c|}{$0.03 M$} & \multicolumn{3}{|c|}{$0.05 M$} \\
\hline & $\Delta K_{\text {ex }}$ & $\Delta K_{\text {theo }}$ & $\%$ Diff. & $\Delta K_{\mathrm{ex}}$ & $\Delta K_{\text {theo }}$ & $\%$ Diff. & $\Delta K_{\text {ex }}$ & $\Delta K_{\text {theo }}$ & $\%$ Diff. \\
\hline 1 & 0.58 & 0.70 & 19 & 0.35 & 0.48 & 31 & 0.24 & 0.36 & 40 \\
\hline 3 & 0.52 & 0.58 & 11 & 0.33 & 0.52 & 45 & 0.23 & 0.41 & 56 \\
\hline 5 & 0.07 & 0.16 & 78 & 0.09 & 0.31 & 110 & 0.00 & 0.31 & 200 \\
\hline
\end{tabular}

TABLE 8

Comparison of Experimental and Theoretical $\Delta K$ for Cascade Polymers in Different Ionic Strength Buffers at pH 7.0 


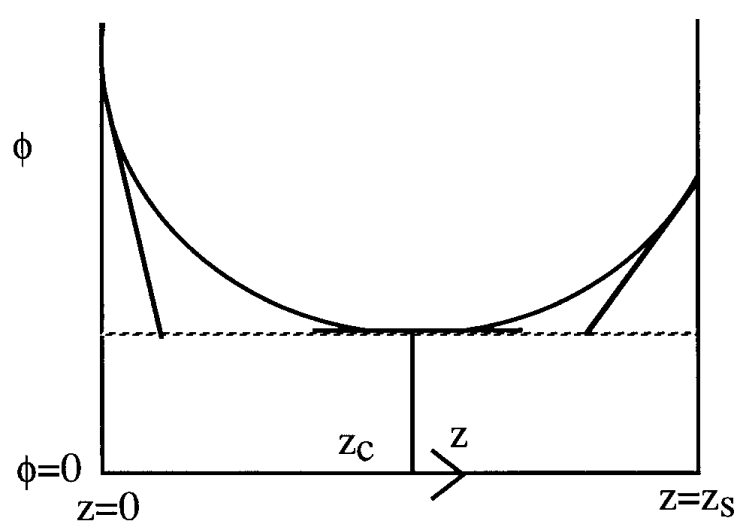

where $z=0$ is the position of the cylinder cavity surface and $z_{\mathrm{s}}$ is the position of the sphere surface. Note that the potential slopes, as determined by the boundary conditions (Eq. [A2], are fixed at $z=0$ and $z=z_{\mathrm{s}}$. The change in the slope from its fixed value at $z=0$ to its value of zero at $z$ $=z_{\mathrm{c}}$ is given for the full Poisson-Boltzmann equation by

$$
2 n_{0} q \sinh (q \phi / k T)
$$

and for the linearized PBE by

$$
2 n_{0} q\left(q \phi_{\mathrm{L}} / k T\right)
$$

Now as the slope must change by a fixed amount on going from the fixed slope at $z=0$ to zero at $z=z_{\mathrm{c}}$, it follows that

$$
2 n_{0} q \sinh (q \phi / k T) \cong 2 n_{0} q\left(q \phi_{\mathrm{L}} / k T\right),
$$

but as

$$
\sinh a>a
$$

it follows that

$$
\phi_{\mathrm{L}}>\phi
$$

\section{ACKNOWLED GMENTS}

This work was supported by NSF Grant CHE 9505953 (PLD). We thank Daniel Robertson of the Facility for Computational Molecular and Biomolecular Science at Indiana University-Purdue University for his assistance with the computer calculations. Further support from the National
Science Foundation (DMR-92-17331; 92-08925) and the Army Office of Research (DAAH 04-93-0048) is also acknowledged (GRK).

\section{REFERENCES}

1. Deen, W. M., and Smith, F. G., III, J. Membr. Sci. 12, 217 (1982).

2. Dubin, P. L., Larter, R. M., Wu, C. J., and Kaplan, J. I., J. Phys. Chem. 94, 7243 (1990).

3. Dubin, P. L., and Tecklenburg, M. M., Anal. Chem. 57, 275 (1985).

4. Dubin, P. L., Speck, C. M., and Kaplan, J. I., Anal. Chem. 60, 895 (1988).

5. Mori, S., Anal. Chem. 61, 530 (1989).

6. Styring, M. G., Davison, C. J., Price, C., and Booth, C., J. Chem. Soc. Faraday Trans 1 80, 3051 (1984).

7. Styring, M. G., Honing, J. A. J., and Hamielec, A. E., J. Liq. Chromatogr. 9, 3505 (1986).

8. Potschka, M., J. Chromatogr. 587, 276 (1991).

9. Potschka, M., J. Chromatogr. 441, 239 (1988).

10. Cai, C.-H., Romano, V., and Dubin, P. L., J. Chromatogr. 693, 251 (1995).

11. Lin, N. P., and Deen, W. M., J. Colloid Interface Sci. 153, 483 (1992).

12. Lin, N. P., and Deen, W. M., Macromolecules 23, 2947 (1990).

13. Hoagland, D. A., Macromolecules 23, 2781 (1990).

14. Smith, F. G., III, and Deen, W. M., J. Colloid Interface Sci. 78, 44 (1980).

15. Smith, F. G., III, and Deen, W. M., J. Colloid Interface Sci. 91, 571 (1983).

16. It is generally conceded, partly on the basis of the absence of flow rate effects, that SEC is an equilibrium process. For discussion, see Casassa, E. F., J. Phys. Chem. 75, 3929 (1971).

17. Dubin, P. L., Edwards, S. L., Kaplan, J. I., Mehta, M. S., Tomalia, D., and Xia, J., Anal. Chem. 64, 2344 (1992).

18. Young, J. K., Baker, G. R., Newkome, G. R., Morris, K. F., and Johnson, Jr., C. S., Macromolecules 27, 3464 (1994).

19. Newkome, G. R., Young, J. K., Baker, G. R., Potter, R. L., Audoly, L., Cooper, C., and Weis, C. D., Macromolecules 26, 2394 (1993).

20. Lavrenko, P. N., Mikryukova, O. I., and Didenko, S. A., Polym. Sci. U.S.S.R. 28, 576 (1986).

21. Haller, W., Nature 206, 693 (1965).

22. Haller, W., Basedow, A. M., König, B. J., J. Chromatogr. 132, 387 (1977).

23. Ouano, A. V., Rubber Chem. Technol. 54, 535 (1981).

24. Waldmann-Meyer, H., J. Chromatogr. 350, 1 (1985).

25. Hiemenz, P. C., "Principles of Colloid Chemistry,' Chap. 9. Dekker, New York, 1977.

26. Hiemenz, P. C., 'Principles of Colloid Chemistry,' Chap. 13. Dekker, New York, 1977.

27. Flory, P. J., 'Principles of Polymer Chemistry,' p. 606. Cornell Univ. Press, Ithaca, 1953.

28. Shah, G., and Dubin, P. L., J. Chromatogr. 693, 197 (1995).

29. Fuoss, R. M., Discuss. Faraday Soc. 11, 125 (1952).

30. Zimm, B. H., and Le Bret, M., J. Biomol. Struct. Dyn. 1, 461 (1983).

31. Potschka, M., J. Chromatogr. 441, 239 (1988).

32. Zu, Y., Cai, S., Dubin, P. L., and Potschka, M., in preparation. 